Article

\title{
Evolution of the Annealing Twin Density during $\delta$-Supersolvus Grain Growth in the Nickel-Based Superalloy Inconel ${ }^{\mathrm{TM}} 718$
}

\author{
Yuan Jin ${ }^{1, *}$, Marc Bernacki ${ }^{1}$, Andrea Agnoli ${ }^{1}$, Brian Lin ${ }^{2}$, Gregory S. Rohrer ${ }^{2}$, \\ Anthony D. Rollett ${ }^{2}$ and Nathalie Bozzolo ${ }^{1, *}$ \\ Received: 1 November 2015; Accepted: 17 December 2015; Published: 24 December 2015 \\ Academic Editor: Johan Moverare \\ 1 MINES ParisTech, PSL—Research University, CEMEF-Centre de mise en forme des matériaux, \\ CNRS UMR 7635, CS 10207 rue Claude Daunesse, Sophia Antipolis Cedex 06904, France; \\ marc.bernacki@mines-paristech.fr (M.B.); andrea.agnoli.job@gmail.com (A.A.) \\ 2 Department of Materials Science and Engineering, Carnegie Mellon University, 5000 Forbes Avenue, \\ Pittsburgh, PA 15213, USA; brianklin@gmail.com (B.L.); gr20@andrew.cmu.edu (G.S.R.); \\ rollett@andrew.cmu.edu (A.D.R.) \\ * Correspondence: jyuan915@gmail.com (Y.J.); nathalie.bozzolo@mines-paristech.fr (N.B.); \\ Tel.: +32-472-851-068 (Y.J.); +33-493-678-945 (N.B.); Fax: +32-719-109-31 (Y.J.); +33-492-389-752 (N.B.)
}

\begin{abstract}
Grain growth experiments were performed on Inconel ${ }^{\mathrm{TM}} 718$ to investigate the possible correlation of the annealing twin density with grain size and with annealing temperature. Those experiments were conducted at different temperatures in the $\delta$ supersolvus domain and under such conditions that only capillarity forces were involved in the grain boundary migration process. In the investigated range, there is a strong inverse correlation of the twin density with the average grain size. On the other hand, the twin density at a given average grain size is not sensitive to annealing temperature. Consistent with previous results for pure nickel, the twin density evolution in Inconel ${ }^{\mathrm{TM}} 718$ is likely to be mainly controlled by the propagation of the pre-existing twins of the growing grains; i.e., the largest ones of the initial microstructure. Almost no new twin boundaries are created during the grain growth process itself. Therefore, the twin density at a given average grain size is mainly dependent on the twin density in the largest grains of the initial microstructure and independent of the temperature at which grains grow. Based on the observations, a mean field model is proposed to predict annealing twin density as a function of grain size during grain growth.
\end{abstract}

Keywords: annealing twin; grain growth; EBSD; mean field model

\section{Introduction}

Nickel-based superalloys are used for aeronautical component manufacturing because of their performance at high temperature. Grain boundary engineering (GBE) is a possible route to improve the properties, especially those related to intergranular damage [1]. Because of their low energy [2], annealing twin boundaries, observed in almost all deformed and subsequently annealed face-centered-cubic (FCC) metals with low to medium stacking fault energy, are fundamental for GBE [3-5]. Even though these crystalline defects have been known for a long time [6], the mechanisms by which they appear are still not fully understood. Being able to predict the twin density obtained after a given thermomechanical path, which has been made on an empirical basis for now, would be very valuable for developing GBE routes.

The growth accident model, which asserts that a coherent twin boundary forms at a migrating grain boundary due to a stacking error, is most commonly used to explain annealing twin 
formation [7-9]. In the growth accident model, the amplitude of the driving force acting on grain boundary migration and the resulting migration velocity are promoting factors for the generation of annealing twins. However, few studies in the literature address the direct effect of the grain boundary migration rate. The aim of the present work is to contribute to filling this gap. Grain growth experiments are performed on a nickel-based superalloy (Inconel ${ }^{\mathrm{TM}} 718$ ) at different temperatures, so that grain boundaries migrate at different rates. The twin content evolution is discussed quantitatively in relation to temperature, grain size increase and grain boundary migration rate. A mean field model is proposed based on the observations to describe the average twin density evolution as a function of grain size during grain growth.

\section{Experimental Details for Quantitative Analysis of Twin Content}

Grain growth experiments were performed on semi-cylindrical samples machined from an Inconel $^{\mathrm{TM}} 718$ billet at three different temperatures above the solvus for the delta phase and for different times (Table 1).

Table 1. Annealing conditions (annealing times for each temperature) applied on the initial microstructure shown in Figure 1 to obtain those shown in Figure 2.

\begin{tabular}{cccc}
\hline Temperature & \multicolumn{3}{c}{ Applied Annealing Time } \\
\hline $1025^{\circ} \mathrm{C}$ & $10 \mathrm{~min}$ & $25 \mathrm{~min}$ & \\
$1065^{\circ} \mathrm{C}$ & $3 \mathrm{~min}$ & $6 \mathrm{~min}$ & $9 \mathrm{~min}$ \\
$1100^{\circ} \mathrm{C}$ & $1 \mathrm{~min}$ & $2 \mathrm{~min}$ & $3 \mathrm{~min}$ \\
\hline
\end{tabular}

All of the analyzed samples (longitudinal section) were metallographically prepared with a prolonged final mechanical polish using a $0.5-\mu \mathrm{m}$ colloidal $\mathrm{SiO}_{2}$ suspension. The electron backscatter diffraction (EBSD) maps were obtained with a ZEISS SUPRA 40 FEG SEM (Jena, Germany) equipped with a Bruker CrystAlign EBSD system (Berlin, Germany). The step size for EBSD map acquisition was set to $0.46 \mu \mathrm{m}$ for the initial microstructure. However, as the average grain size in the annealed samples became larger, the step size was accordingly increased to $1.44 \mu \mathrm{m}$, which appeared as a good compromise for measuring a sufficient number of grains, but still completing each map within a reasonable acquisition time. The mapped area of the initial microstructure contains more than 5000 grains. The EBSD maps recorded after grain growth include 150 to 800 grains (twins being excluded in grain counting). The OIM ${ }^{\mathrm{TM}}$ software (EDAX, Mahwah, NJ, USA) was used to analyze the EBSD data.

Grain boundaries were defined as boundaries with a misorientation angle above $5^{\circ}$. Annealing twin boundaries are defined by a misorientation of $60^{\circ}$ about the $<111>$ axis with a tolerance of $8.66^{\circ}$, according to Brandon's criterion [10], regardless of their coherent versus incoherent character. The Brandon's criterion was applied because an F.C.C. twin can also be depicted as an $\Sigma 3$ coincidence site lattice. Two different quantities were used to quantify annealing twins: the annealing twin density $\left(N_{\mathrm{L}}\right)$ and the number of annealing twin boundaries per grain $\left(N_{\mathrm{G}}\right)$, respectively defined as:

$$
\begin{aligned}
N_{\mathrm{L}} & =\frac{L_{\mathrm{tb}}}{S} \times \frac{2}{\pi} \\
N_{\mathrm{G}} & =\frac{N_{2}-N_{1}}{N_{1}}
\end{aligned}
$$

where $L_{\mathrm{tb}}$ is the twin boundary length detected in a given sample section area $S, N_{1}$ is the number of grains ignoring twin boundaries in the grain detection procedure and $N_{2}$ is the number of grains by considering twin boundaries as grain boundaries. The formulation to calculate the twin density $\left(N_{\mathrm{L}}\right)$ is derived from [11].

The recrystallized grains were identified in the EBSD maps using a criterion that the grain orientation spread (GOS) was less than $1^{\circ}$ [12]. Twin boundaries were ignored in the grain detection 
procedure (except for determining the $N_{2}$ value), and grains with an area smaller than three pixels were not considered. No additional clean-up was performed. The number-weighted average grain diameter $(D)$ was used to quantify average grain size (equivalent grain diameter) in the following analyses.

\section{Experimental Results and Discussion of the Underlying Mechanisms}

The initial microstructure (Figure 1a) is almost fully recrystallized (more than 93\%) as indicated by the very low GOS values (Figure 1b). The average size of the recrystallized grains is $13 \mu \mathrm{m}$. As depicted by the dashed line in Figure 1d, the twin density in the initial microstructure first increases with grain size, is maximal for grains of about the average grain size and then slowly decreases at larger grain sizes. The twin density in the grains of a given grain size class is calculated with the twin boundary length in these grains and the surface occupied by these grains. This initial microstructure results from the prior recrystallization process. It has been established that the twin density after recrystallization is mainly controlled by the stored energy level $[13,14]$ and that the twin formation event occurs more often when the grains are growing faster $[9,15]$. The migration rate of the recrystallization front decreases during the recrystallization process because the remaining stored energy level in the deformed matrix is decreasing. Therefore, the growth rate of a recrystallized grain decreases, and fewer twins are formed. Consequently, the twin density decreases with increasing the size of the recrystallized grains. The reason why small grains also have a lower twin density is different. The small grains observed in a 2D section after complete recrystallization are either truly small (in 3D) and have a nucleated late in the recrystallization process or they are sections near the edge of a large grain. In both cases, they correspond to a volume that has been swept late, and thus, relatively slowly, by the recrystallization front because of the low level of remaining stored energy.

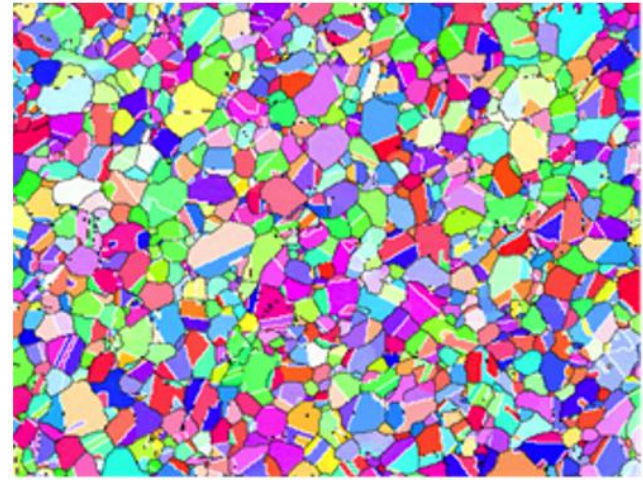

(a)

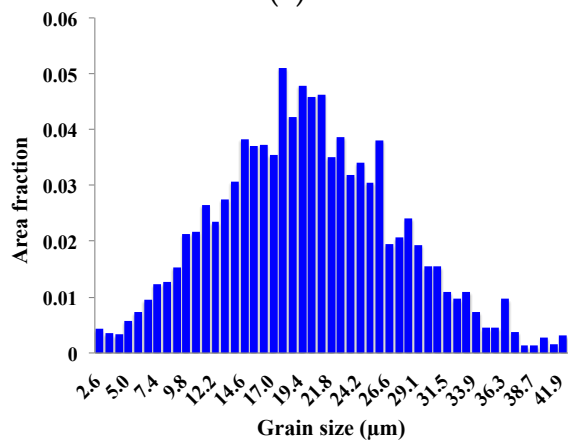

(c)

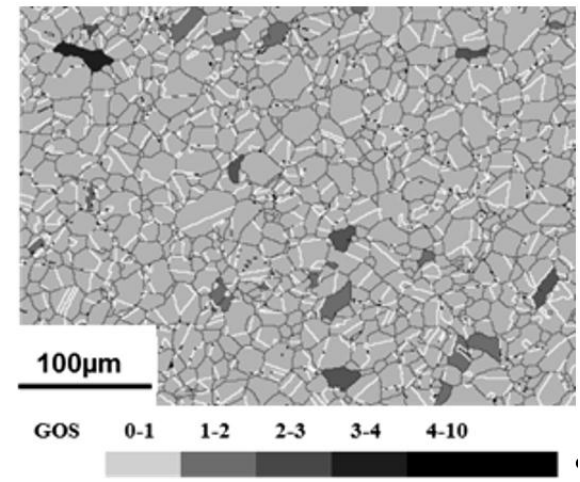

(b)

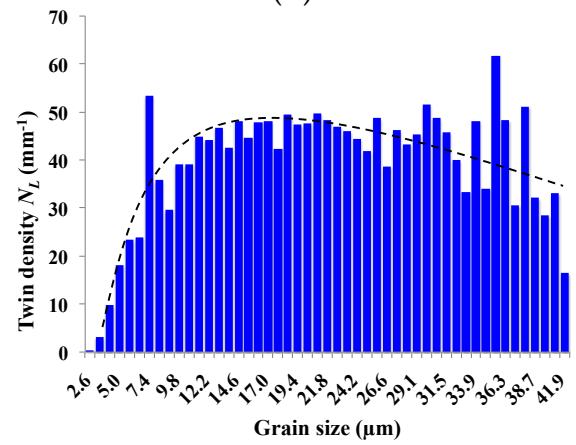

(d)

Figure 1. The initial microstructure: (a) orientation color-coded EBSD map (vertical direction of the map projected in the standard triangle); (b) grain orientation spread (GOS) map; grain boundaries in black and twin boundaries in white; (c) grain size distribution histogram (average grain size $=13 \mu \mathrm{m}$ ); (d) twin density as a function of grain size; the dashed line is a tendency guideline. 
The microstructure evolution at the three different annealing temperatures is shown in Figure 2. Grain growth kinetics are described in Figure 3. As expected, grain growth is faster when increasing the annealing temperature. The annealing times were adapted accordingly to result in similar grain sizes (in each column of Figure 2).
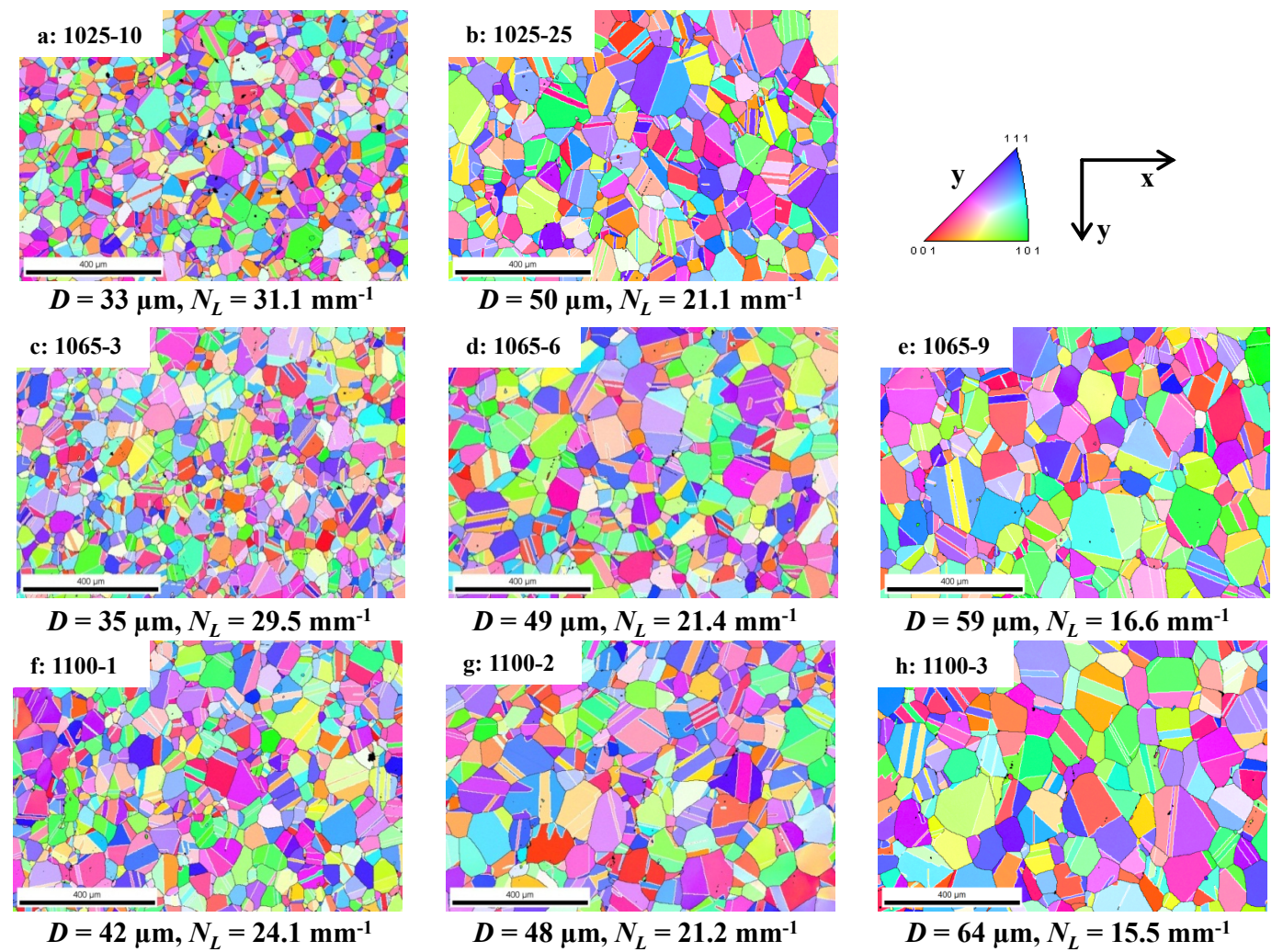

Figure 2. Microstructure evolution after annealing (same color code as in Figure 1a). (a) at $1025^{\circ} \mathrm{C}$ for $10 \mathrm{~min},(\mathbf{b})$ at $1025^{\circ} \mathrm{C}$ for $25 \mathrm{~min}$, (c) at $1065^{\circ} \mathrm{C}$ for $3 \mathrm{~min}$, (d) at $1065^{\circ} \mathrm{C}$ for $6 \mathrm{~min}$, (e) at $1065^{\circ} \mathrm{C}$ for $9 \mathrm{~min},(\mathbf{f})$ at $1100{ }^{\circ} \mathrm{C}$ for $1 \mathrm{~min},(\mathrm{~g})$ at $1100{ }^{\circ} \mathrm{C}$ for $2 \mathrm{~min},(\mathbf{h})$ at $1100{ }^{\circ} \mathrm{C}$ for $3 \mathrm{~min}$. The corresponding average grain size and annealing twin density are specified under each map. The white lines denote twin boundaries; the black lines are grain boundaries with a disorientation greater than $5^{\circ}$.

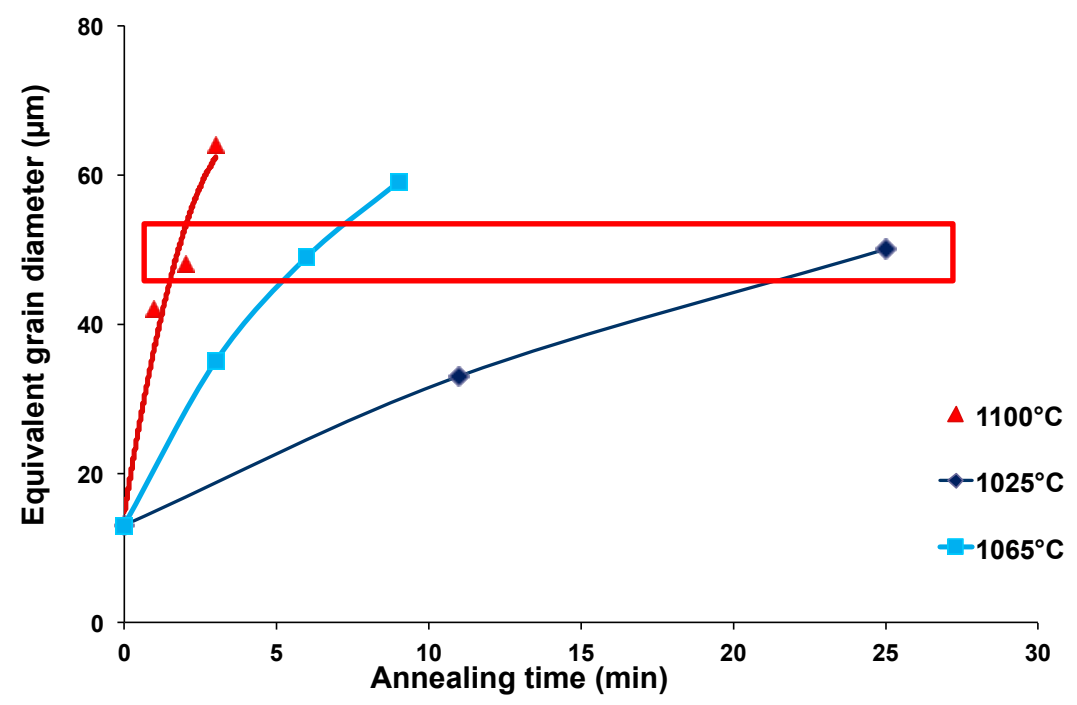

Figure 3. Grain growth kinetics at different annealing temperatures. 
The twin density is plotted as a function of the average grain size in the whole microstructure in Figure 4. The total twin boundary length and the surface of the overall microstructure are used to calculate the overall twin density via Equation (1). There is a strong inverse correlation of the twin density with the average grain size; similar trends have already been reported in the literature for different FCC metals and alloys [7,15-18].

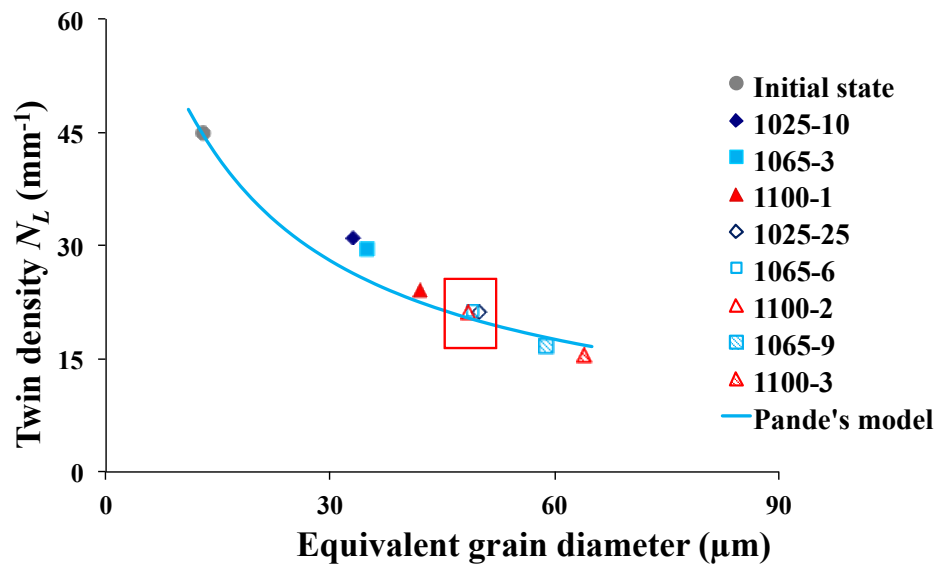

Figure 4. Twin density evolution compared to Pande's model (best fit to Equation (3) with $\gamma_{\mathrm{gb}} \approx 1 \mathrm{~J} \cdot \mathrm{m}^{-2}$ ).

According to Pande [18], the annealing twin evolution depends on the grain boundary migration driving force and on the grain boundary migration distance (and therefore, on grain size). In order to test the influence of grain size on annealing twin development, microstructures with an identical average grain size of about $50 \mu \mathrm{m}$, but obtained at different annealing times and temperatures, were compared (highlighted by the red rectangle in Figures 3 and 4). Despite the difference in grain growth kinetics at the three temperatures, the twin densities for the same average grain size $(50 \mu \mathrm{m})$ are identical. Therefore, the twin density evolution appears to be independent of grain growth kinetics within the considered range. This observation is consistent with experimental data reported in the early literature [19].

In addition to the twin density, the number of annealing twin boundaries per grain $\left(N_{\mathrm{G}}\right)$ was also used for annealing twin quantification. $N_{\mathrm{G}}$ is calculated using the total grain numbers $N_{2}$ with and $N_{1}$ without considering twin boundaries as grain boundaries in the overall microstructure. In order to determine the density of twins formed during a given process, i.e., to describe the occurrence of twin formation events, the number of twin boundaries per developing grain provides a better indicator than any of the other quantification parameters. During mean curvature-driven grain growth, the bigger grains grow at the expense of the smaller ones. Therefore, twin formation in the largest grains (about 100 grains) in each analyzed sample was investigated. In the sample with the largest average grain size (1100-3), the 105 largest grains occupied more than $95 \%$ of the EBSD mapped area (Figure 5). The number of twin boundaries per grain in the largest grains does not increase during grain growth (Figure 6); if anything, it decreases, although the counts are not large enough to have high confidence in this conclusion. This observation is consistent with the results of in situ annealing on pure nickel reported in [15]. Indeed, in this previous work, most annealing twins were formed during recrystallization, and very few new twins were observed during grain growth. In the grain growth regime, small grains containing several twin boundaries are consumed by large grains, which grow, but only very rarely produce new twins. A new mesoscopic model, which relates annealing twin formation to the topology of the moving grain boundaries, was proposed in $[13,15,16]$ to explain this phenomenon. According to this model, coherent twins can nucleate only along grain boundary segments that migrate opposite to their curvature. During grain growth, this may happen only at triple junctions: in other words, only triple junctions can be potential nucleation sites for annealing twins. This idea was confirmed by a recent 3D in situ study [20]. In this study, 
the near-field high-energy X-ray diffraction microscopy (nf-HEDM) technique was used to follow the microstructure evolution during grain growth in a pure nickel sample. In this 3D experiment, only very few twin formation events were observed and all of them were at triple junctions.

The fact that the large grains develop almost without creating new twins, allied with the consumption of the twinned small grains, contributes to the inverse correlation of the twin density with the increasing average grain size in the work on Inconel ${ }^{\mathrm{TM}} 718$ shown in Figure 4 . Furthermore, incoherent twin boundaries may migrate in a direction that reduces twin length and total interfacial energy, which is an additional reason why the twin density decreases during grain growth (it is worth mentioning here that this additional mechanism is possibly material and temperature dependent). In addition, the fact that the twin evolution is independent of grain growth kinetics is consistent with the above-described mechanism where twin density evolution is mainly controlled by the extension of pre-existing twins (those formed during prior recrystallization and existing in the large grains at the beginning of the grain growth regime). How much the twins extend depends on how much the grains grow, but not on how fast they grow.

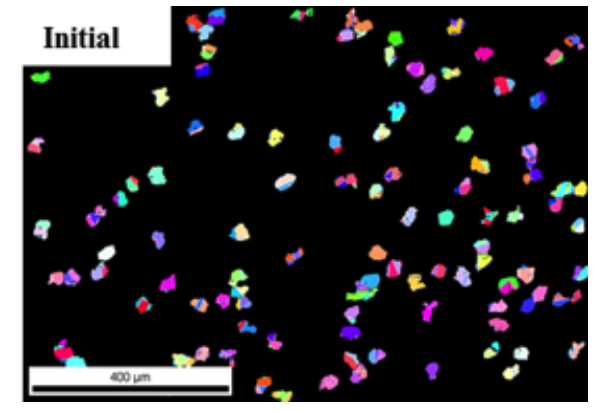

(a)

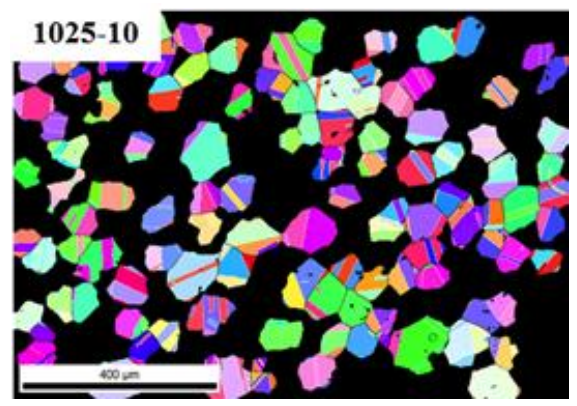

(b)

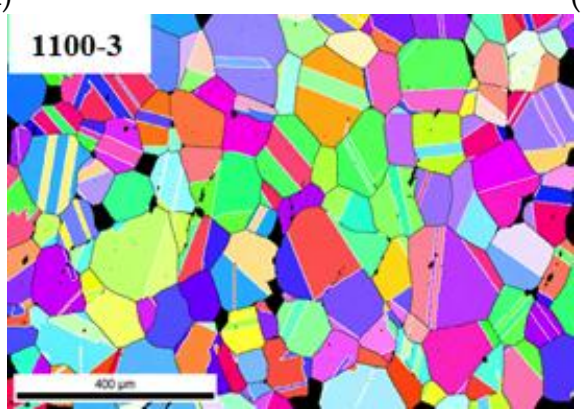

(c)

Figure 5. The (about 100) largest grains in (a) the initial microstructure, (b) the sample annealed at $1025^{\circ} \mathrm{C}$ for $10 \mathrm{~min}$ and (c) the sample annealed at $1100{ }^{\circ} \mathrm{C}$ for $3 \mathrm{~min}$. Same color code as in Figure 1.

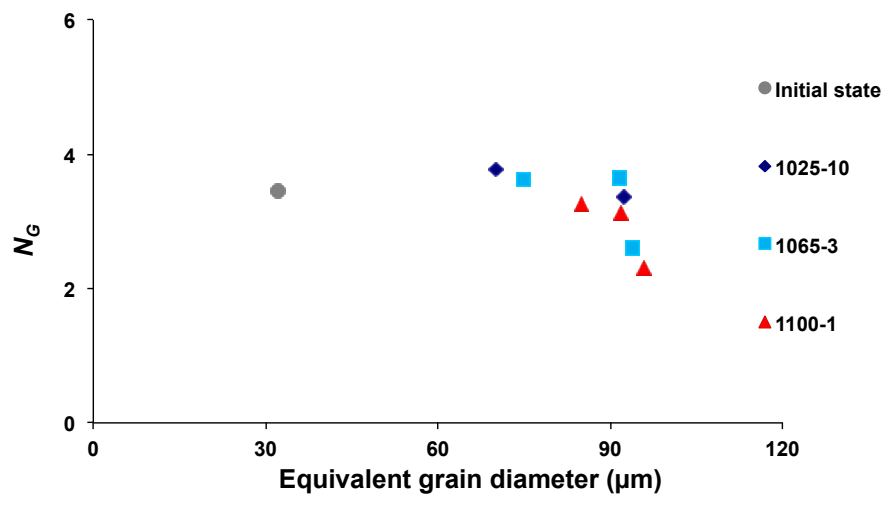

Figure 6. Number of twin boundaries per grain in the 100 largest grains expressed as a function of the average grain size. 


\section{Literature Models for Predicting Twin Density Evolution}

Two main mathematical models to predict twin density exist in the literature, namely Gleiter's model [21] and Pande's model [18], which are both consistent with the growth accident theory. In Gleiter's model, derived from classical nucleation theory, the twin formation probability mainly depends on two factors: the activation enthalpy for migration and the grain boundary migration driving force. However, it is shown in [16] that contrary to the experimental results described in this study and in other works in the literature [19], the twin density predicted by this model is very sensitive to temperature. Meanwhile, Pande's model is shown to be consistent with various experimental data $[7,17,18]$. Consequently, Pande's model is most often used to compare to the experimental data in the literature. Originally formulated to consider curvature-driven growth, Pande's model has also been adapted to account for the stored energy contribution to the grain boundary migration driving force [7].

Pande's model [18] is derived from the assumption that the increment of annealing twin boundary number per grain $\left(\Delta N_{G}\right)$ is proportional to the grain boundary migration driving force $(F)$ and the increase in grain size $(\Delta D)$. Therefore, the model assumes that new twins are formed $\left(N_{\mathrm{G}}\right.$ increasing) while grains grow ( $D$ increasing). This is not consistent with our observations within the grain growth regime, presented here for Inconel ${ }^{\mathrm{TM}} 718$ and in the previous paper for pure nickel [15].

The correlation between the annealing twin density and the average grain size can nevertheless be fitted quite well (see Figure 4) by the formula derived from Pande's model (Equation (3)) [18]:

$$
P=\frac{1}{D} K \gamma_{\mathrm{gb}} \ln \frac{D}{D_{0}}
$$

with $\gamma_{\mathrm{gb}} \approx 1 \mathrm{~J} \cdot \mathrm{m}^{-2}, K \approx 0.3 \mathrm{~m}^{3} \cdot \mathrm{J}^{-1}$ and $D_{0} \approx 2 \mu \mathrm{m}$, where the parameter values were identified based on experimental data (Figure 4) by an inverse method. A MATLAB function "fminsearch" was used to identify the parameters that minimize the cost function, which is defined as the sum of the squared residuals. A residual is defined here as the difference between a value in the experimental data and the corresponding value predicted by Pande's model. Even though Pande's model can indeed describe the correlation between the annealing twin density and the average grain size, the underlying modeling assumptions are not consistent with the experimental observation of annealing twin evolution. Accordingly, a mean field model will be attempted in the next section to address this gap.

\section{Description of the New Mean Field Model}

As presented in the Section 3, there are almost no annealing twins formed in the grain growth regime. This is the basis statement for the new model proposed here that aims at describing the average twin density evolution in the overall microstructure as a function of the average grain size during grain growth.

In the present study and for most of the experimental data in the literature, twin boundaries are quantified in 2D. Accordingly, for the sake of brevity, only the 2D version of the mean field model is presented here. The possibility of the 3D extension will be discussed in Section 7.

The microstructure is discretized into $n$ categories of representative grains $G_{i}, i \in\{1, \ldots, n\}$, based on the same principle applied by [22], but here, representative grains are defined by two variables: the grain diameter $D_{i}$ and the assumed related twin boundary length $L_{\mathrm{tb}}^{i}$. Representative grains $G_{i}$ stand for the average state of a number $N_{i}$ of assumed identical (in terms of diameter and, so, twin boundary length) circular grains present in all of the microstructure.

In mean curvature-driven grain growth with isotropic mobility and grain boundary energy (which can be assumed since twin boundaries are not considered as grain boundaries, but as internal defects of the growing grains), the two-dimensional average growth rate of the representative grain 
$G_{i}$, i.e., of the $N_{i}$ grains belonging to the $i$-th category, can be approximated using Hillert's classical mean field model [23]:

$$
\frac{\mathrm{d} D_{i}}{\mathrm{~d} t}=2 \cdot M \gamma_{\mathrm{gb}}\left(\frac{1}{D}-\frac{1}{D_{i}}\right)
$$

where $M$ is the grain boundary mobility and $D$ is the average grain size of the microstructure.

In this context, the representative grains that are larger than the average size grow, and those smaller than the average size shrink. This deterministic growth rate thus represents the average behavior of large populations of grains.

As presented previously, essentially no twins formed during grain growth; thus, the twin evolution is mainly controlled by the evolution of the pre-existing twins. More concretely, annealing twin boundaries intersecting grain boundaries are extended or reduced in length as the grain boundary migrates. The basic principle of the model is to describe by how much the twin length in a grain changes when the grain size either increases or decreases. The real topology of twin boundaries can be complex [24], especially that of incoherent twins, which obviously differ from coherent ones [25]. It would thus be quite complicated, if not impossible, to derive a proper analytical description of the change in twin boundary length associated with a change in grain size. Instead, we have tested a rough, but simple assumption, which is as follows. The change in twin boundary length $L_{\mathrm{tb}}^{i}$ is considered as proportional to the change in grain size $D_{i} \forall i \in\{1, \ldots, n\}$ and in each time increment.

In mathematical terms, the assumptions of the model become:

$$
\frac{L_{\mathrm{tb}}^{i}(t+\Delta t)-L_{\mathrm{tb}}^{i}(t)}{L_{\mathrm{tb}}^{i}(t)}=k \frac{D_{i}(t+\Delta t)-D_{i}(t)}{D_{i}(t)}, \forall i \in\{1, \ldots, n\}
$$

In the first approximation, $k$ will be assumed to be constant, i.e., identical for all of the categories and identical for growing grains and shrinking grains, which again are strong assumptions.

When a grain category $G_{k}$ is fully consumed by other categories, i.e., $D_{k}(t+\Delta t)=0$, its corresponding twin boundary length, $L_{\mathrm{tb}}^{k}(t+\Delta t)$ is fixed to zero (which is not automatically obtained using Equation (5) for $k \neq 1$ ). For idealized circular grains, the proportionality factor $k$ seems mainly influenced by two factors, illustrated in Figure 7:

- For a coherent twin boundary spanning to the opposite side of the representative grain, since $\Delta L_{\mathrm{tb}}^{i} \geqslant \Delta D_{i}$ (equality occurs when $L_{\mathrm{tb}}^{i}$ is a diameter of the considered circular representative grains) and $L_{\mathrm{tb}}^{i} \leqslant D_{i}$, we have $\Delta L_{\mathrm{tb}}^{i} / L_{\mathrm{tb}}^{i} \geqslant \Delta D_{i} / D_{i} ;$ thus $k$ should in principle be higher than one. However, if there is more than one coherent twin boundary inside the representative grain, the total twin boundary length is not necessarily smaller than the representative grain diameter. In this case, the value of $k$ depends also on the initial ratio between the twin boundary length and the representative grain diameter.

- Incoherent twin boundary segments may migrate inside representative grains to decrease the total twin boundary length. This may lead to the shortening of the considered twin boundary, even though the representative grain boundary migration tends to lengthen it, which is a reason for which $k$ might be smaller than one.

The possible material and temperature dependencies of incoherent twin boundary migration behavior are implicitly considered in the parameter $k$. In the following, the constant $k$ will be identified based on the experimental results presented in Section 3. The algorithm of the mean field model can be summarized as follows.

During a time step:

- The diameter change of each representative grain is calculated using Equation (4). The volume conservation is naturally verified. 
- The change in twin length for each category $N_{i} \Delta L_{\mathrm{tb}}^{i}$ is calculated by Equation (5) using the twin boundary length in this category $N_{i} L_{\mathrm{tb}}^{i}$, since $N_{i} \Delta L_{\mathrm{tb}}^{i} / N_{i} L_{\mathrm{tb}}^{i}=\Delta L_{\mathrm{tb}}^{i} / L_{\mathrm{tb}}^{i}$ (when a grain category $G_{i}$ is fully consumed by other categories, i.e., $D_{i}=0$, its corresponding twin boundary length, $L_{\mathrm{tb}}^{i}$, is fixed to zero).

- The twin density in each category $\left(N_{\mathrm{L}}^{i}\right)$ is calculated via the twin boundary length in this category $\left(N_{i} L_{\mathrm{tb}}^{i}\right)$ and its area $\left(N_{i} S_{i}\right)$ as follows:

$$
N_{\mathrm{L}}^{i}=\frac{2}{\pi} \cdot \frac{N_{i} L_{\mathrm{tb}}^{i}}{\pi N_{i}\left(\frac{D_{i}}{2}\right)^{2}}=\frac{2}{\pi} \cdot \frac{N_{i} L_{\mathrm{tb}}^{i}}{N_{i} S_{i}}
$$

- The average twin density in the overall microstructure is calculated from the summation of twin boundary lengths of each category and the overall area $(S)$ as follows:

$$
N_{\mathrm{L}}=\frac{2}{\pi} \cdot \frac{\sum_{i} N_{i} L_{\mathrm{tb}}^{i}}{\sum_{i} N_{i} S_{i}}=\frac{2}{\pi} \cdot \frac{\sum_{i} N_{i} L_{\mathrm{tb}}^{i}}{S}
$$

Taken together, to predict the evolution of the average twin density, we need a grain size distribution with the area and the twin boundary length of each category as the input.
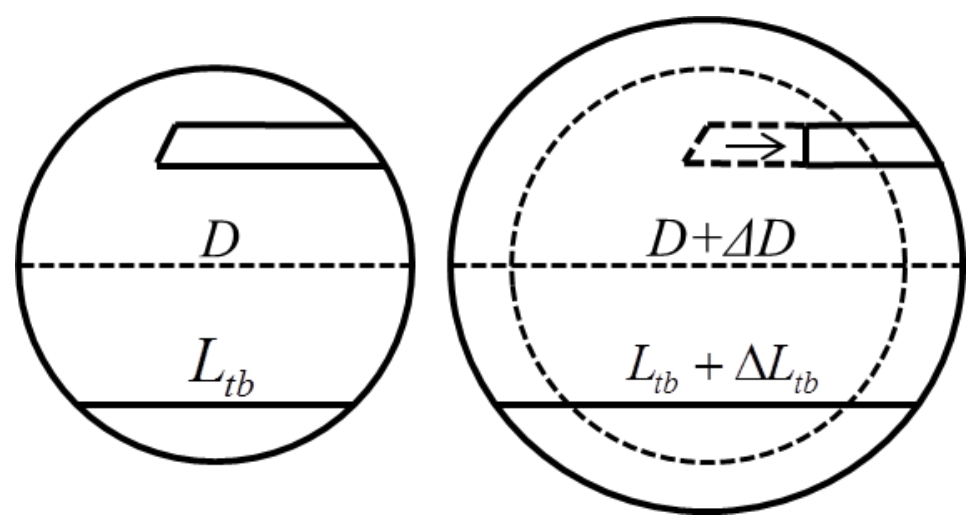

Figure 7. Annealing twin evolution mechanisms during grain growth.

\section{Mean Field Modeling of Twin Density Evolution during Grain Growth in Inconel ${ }^{\mathrm{TM}} \mathbf{7 1 8}$}

The grain size distribution and the twin density in each grain size class of the initial microstructure of Inconel ${ }^{\mathrm{TM}} 718$, shown in Figure 1c,d, was used as input. The EBSD data were discretized into 49 grain size categories (Figure 1c), and the twin density was measured for each category (Figure 1d).

With regard to the physical parameters of the model, the grain boundary mobility $M$ and the grain boundary energy $\gamma_{\mathrm{gb}}$ values were chosen within the range of typical values for metals $\left(M \gamma_{\mathrm{gb}}=8.22 \times 10^{-13} \mathrm{~m}^{2} / \mathrm{s}\right)$. This value therefore does not refer to a specific material. Since this model aims at predicting the twin density evolution as a function of grain size, but not as a function of time, it is not necessary to stick to the real grain growth kinetics of a particular material. Furthermore, for a given material, the twin density evolution is not influenced by the grain growth kinetics (within the present modeling assumptions and consistent with our experimental observations).

The average annealing twin density evolution in the overall microstructure modeled by the mean field model with the time step $\mathrm{d} t=1 \mathrm{~s}$ is compared in Figure 8 to the Inconel ${ }^{\mathrm{TM}} 718$ grain growth experimental data. The value of 0.9 for $k$ was determined as providing the best fit between the model 
results and the experimental data. With that value of $k$, the present experimental data can be well described by the new mean field model, which thus sounds promising. Additional experimental data would be relevant to fill the gap between the initial grain size $(13 \mu \mathrm{m})$ and $30 \mu \mathrm{m}$. This work on Inconel $^{\mathrm{TM}} 718$ will be completed in the future; and the model will be compared to other experimental grain growth data for other materials.

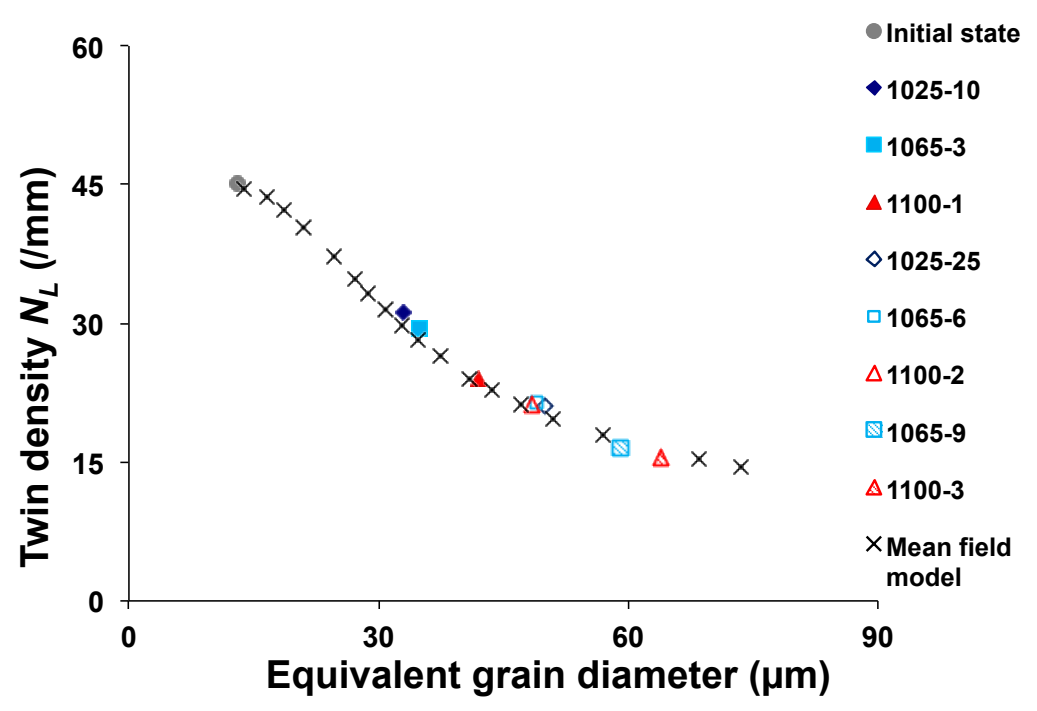

Figure 8. Average twin density $\left(N_{\mathrm{L}}\right)$ evolution compared to the new mean field model.

In addition, for the sake of determining the possible impact of the time step on the modeling results, the evolution of the grain size and twin density distributions at different stages of the mean field modeling using $\mathrm{d} t=1 \mathrm{~s}$ and $0.5 \mathrm{~s}$ are shown in Figure 9. The modeling results using these two time steps are almost identical, which shows the convergence of the calculation.

During the modeling process, small grains are consumed by large grains. The disappearance of small representative grains leads to a decrease in the total number of representative grains, as illustrated by Figure 9 (the decrease in grain category number). The area fraction of the $i$-th category is calculated by the ratio between $N_{i} S_{i}$ and $S$.

In Figure 9, the twin density related to the large representative grains is quite homogenously distributed over the whole range of grain sizes and slightly decreasing as it was in the initial microstructure (Figure 1d). However, the twin density related to the smallest representative grain is much higher. According to Equation (6), for each representative grain, the twin density is inversely correlated to the square of the corresponding grain size. At the same time, the change in twin boundary length $L_{\mathrm{tb}}^{i}$ is proportional to the change in grain size $D_{i}$ in each time increment, as indicated in Equation (5). For the smallest representative grain, consumed by the other grain families, its grain size shrinks much compared to its initial grain size. Therefore, the high twin density related to the smallest representative grain is numerically caused by the substantial decrease in its size. For example, in Figure 9, at $t=3150 \mathrm{~s}$, the grain size of the smallest representative grain is $14.7 \mu \mathrm{m}$, and the related twin density is about $90 \mathrm{~mm}^{-1}$. At $t=375 \mathrm{~s}$, the grain size of the corresponding grain family (marked in Figure 9a) is about $47 \mu \mathrm{m}$, and the related twin density is about $26 \mathrm{~mm}^{-1}$. The behavior of the smallest grain size category is physically controlled by the shrinkage of pre-existing grains. This apparently odd behavior of the model for the smallest grains may be due to one of the model assumptions that $k$ has the same value for growing and shrinking grains. 

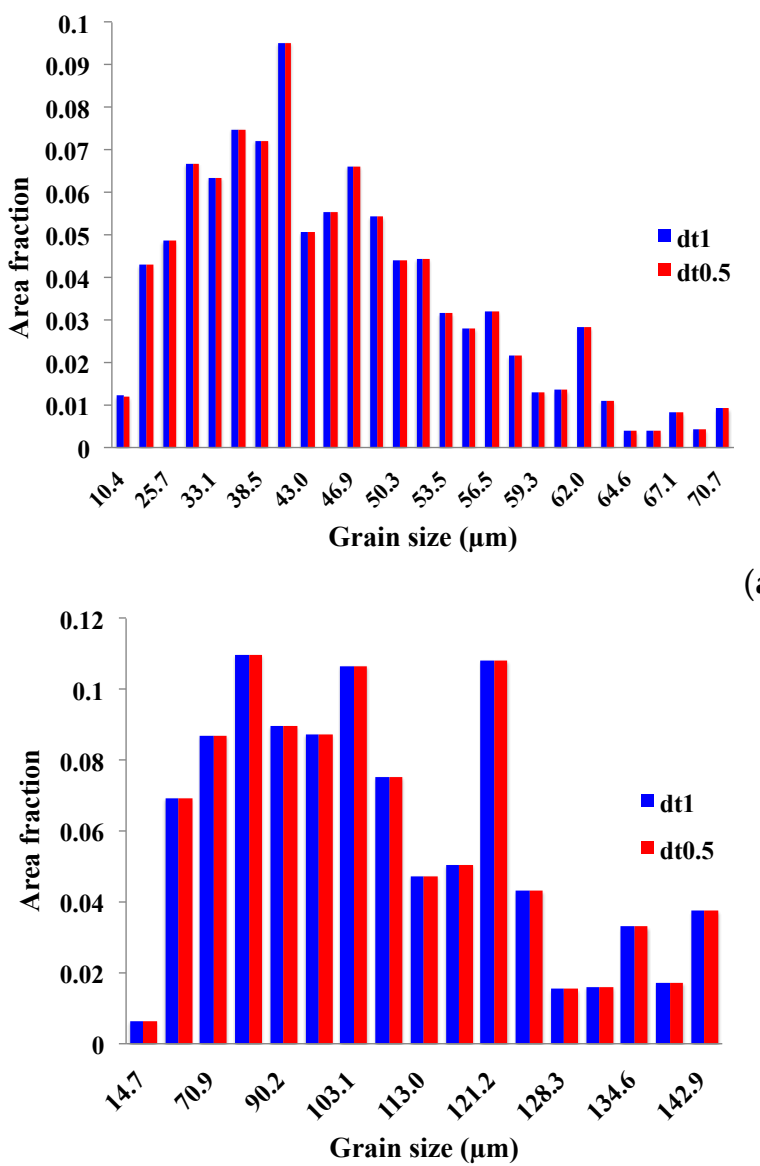

(b)

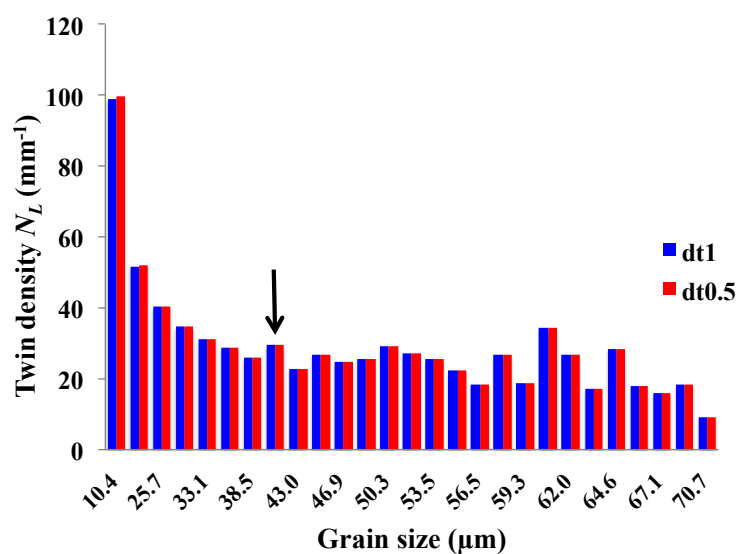

(a)

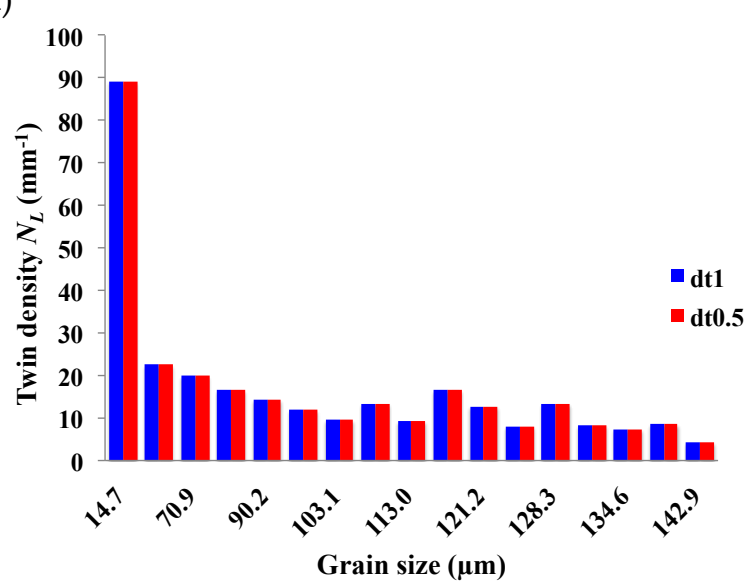

Figure 9. Mean field modelling result at (a) $t=375 \mathrm{~s}$ (average grain size $=32.7 \mu \mathrm{m}$ ) and (b) $t=3150 \mathrm{~s}$ (average grain size $=73.6 \mu \mathrm{m}$ ) obtained using two different time steps $(\mathrm{d} t)$, i.e., one and 0.5. Left: Grain size distributions; right: twin density as a function of grain size. The grain family at $t=375 \mathrm{~s}$ corresponding to the smallest representative grain at $t=3150 \mathrm{~s}$ is pointed out by the black arrow in (a). (a) Mean field modeling results at $t=375 \mathrm{~s}$ obtained using two different time steps $(\mathrm{d} t)$, i.e., one and 0.5 ; (b) mean field modelling results at $t=3150 \mathrm{~s}$.

\section{Discussion of the Relevance of the New Mean Field Model}

The model proposed here accounts for the observed mechanism of annealing twin evolution during grain growth: basically, no new twins are formed, but only the pre-existing ones evolve (either by expanding or shortening while the related grain boundary moves or shortening if an incoherent segment migrates). Despite the assumptions made in the model for the dependence of the twin length in a grain on the size of that grain (simple proportionality), the experimental data (grain growth in Inconel ${ }^{\mathrm{TM}} 718$ ) could be well described by the model. The most remarkable point is that there is only one parameter to be identified, contrary to the other existing models, which have at least two parameters. On the other hand, the new model requires a richer description of the input microstructure (twin length per grain in addition to grain size).

For the sake of comparison, Pande's model has questionable assumptions with regards to the underlying physical mechanisms. Moreover, two parameters must be identified, and this model cannot fit the obtained experimental data as well as the proposed new mean field model (Figure 4 vs. Figure 8).

The model proposed here appears then to be promising, even though it needs to be further validated by comparison with more experimental data. Possible improvements would be to check 
whether a unique value of $k$ can really describe the behavior of all types of grains and to check how far the proportionality relationship of Equation (5) is valid. Both issues are non-trivial, but could be investigated using either 3D microscopy, for example near-field HEDM [20], or by generating synthetic microstructures with a controlled initial twin topology and then evolving them with a full field numerical approach, for example in a finite element context, as already implemented for grain growth in a level-set framework [26-28].

Furthermore, a 3D extension of the present mean field model could be considered, but will require 3D data as an input. The two-dimensional grain size distribution can be reasonably converted to an approached three-dimensional distribution [29]. However, the two-dimensional annealing twin boundary content cannot easily be converted to three-dimensional data. The application of the mean field model on the 3D dataset in [20] will be considered as a perspective of the present work.

\section{Conclusions}

Grain growth experiments were performed at different temperatures on Inconel ${ }^{\mathrm{TM}} 718$ to study the influence of grain growth rate on annealing twin density during grain growth and led to the following conclusions:

1. Annealing twin density evolution in Inconel ${ }^{\mathrm{TM}} 718$ during grain growth is independent of temperature and, thus, also of the average grain growth rate, in the observed temperature range (1025 to $\left.1100^{\circ} \mathrm{C}\right)$.

2. Annealing twin density exhibits an inverse correlation with the average grain size.

3. The number of annealing twin boundaries per grain does not increase with the average grain size. This observation, which is consistent with our previous results in pure nickel, suggests that almost no new twins are formed during grain growth. On the contrary, it may slightly decrease, notably due to the migration of incoherent twin boundary segments.

4. A mean field model with only one parameter to be determined is proposed to predict twin density evolution as a function of grain size during grain growth, based on the observed behavior of annealing twins during grain growth.

Acknowledgments: This work was funded by the French National Research Agency (ANR project called FORMATING: ANR-11-NS09-001-01) and the Materials World Network of the U.S. National Science Foundation under Grant Number DMR-1107986.

Author Contributions: Y.J. performed the experiments and wrote the paper. A.A. helped the set-up of the experiment. N.B. proposed the idea of the mean field model and supervised the work with M.B. and A.D.R. All results were discussed with G.S.R. and B.L.

Conflicts of Interest: The authors declare no conflict of interest.

\section{References}

1. Wantanabe, T. Grain boundary design and control. Res. Mech. 1984, 11, 47-84.

2. Olmsted, D.L.; Foiles, S.M.; Holm, E.A. Survey of computed grain boundary properties in face-centered cubic metals: I. Grain boundary energy. Acta Mater. 2009, 57, 3694-3703. [CrossRef]

3. Palumbo, G.; Lehockey, E.M.; Lin, P. Applications for grain boundary engineered materials. JOM 1998, 50, 40-43. [CrossRef]

4. Randle, V. Twinning-related grain boundary engineering. Acta Mater. 2004, 52, 4067-4081. [CrossRef]

5. Kumar, M.; Schwartz, A.J.; King, W.E. Microstructural evolution during grain boundary engineering of low to medium stacking fault energy fcc materials. Acta Mater. 2002, 50, 2599-2612. [CrossRef]

6. Carpenter, H.; Tamura, S. The formation of twinned metallic crystals. Proc. R. Soc. Lond. A 1926, 113, 161-182. [CrossRef]

7. Cahoon, J.R.; Li, Q.; Richards, N.L. Microstructural and processing factors influencing the formation of annealing twins. Mater. Sci. Eng. A 2009, 526, 56-61. [CrossRef]

8. Li, Q.; Cahoon, J.R.; Richards, N.L. On the calculation of annealing twin density. Scr. Mater. 2006, 55, 1155-1158. [CrossRef] 
9. Wang, W.; Lartigue-Korinek, S.; Brisset, F.; Helbert, A.L.; Bourgon, J.; Baudin, T. Formation of annealing twin during primary recrystallization of two low stacking fault energy Ni-based alloys. J. Mater. Sci. 2015, 50, 2167-2177. [CrossRef]

10. Brandon, D.G. The structure of high-angle grain boundaries. Acta Metall. 1966, 14, 1479-1484. [CrossRef]

11. Underwood, E.E. Quantitative Stereology; Addison-Wesley Publishing Company: Boston, MA, USA, 1970.

12. Alvi, M.H.; Cheong, S.W.; Suni, J.P.; Weiland, H.; Rollett, A.D. Cube texture in hot-rolled aluminum alloy 1050 (AA1050)—Nucleation and growth behavior. Acta Mater. 2008, 56, 3098-3108. [CrossRef]

13. Jin, Y.; Lin, B.; Rollett, A.D.; Rohrer, G.S.; Bernacki, M.; Bozzolo, N. Thermo-mechanical factors influencing annealing twin development in nickel during recrystallization. J. Mater. Sci. 2015, 50, 5191-5203. [CrossRef]

14. Wang, W.; Brisset, F.; Helbert, A.L.; Solas, D.; Drouelle, I.; Mathon, M.H.; Baudin, T. Influence of stored energy on twin formation during primary recrystallization. Mater. Sci. Eng. A 2014, 589, 112-118. [CrossRef]

15. Jin, Y.; Lin, B.; Bernacki, M.; Rohrer, G.S.; Rollett, A.D.; Bozzolo, N. Annealing twin development during recrystallization and grain growth in pure nickel. Mater. Sci. Eng. A 2014, 597, 295-303. [CrossRef]

16. Jin, Y. Annealing Twin Formation Mechanism. Ph.D. Thesis, Mines-ParisTech, Paris, France, December 2014.

17. Detrois, M.; Goetz, R.L.; Helmink, R.C.; Tin, S. Modeling the effect of thermal-mechanical processing parameters on the density and length fraction of twin boundaries in Ni-base superalloy RR1000. Mater. Sci. Eng. A 2015, 647, 157-162. [CrossRef]

18. Pande, C.S.; Imam, M.A.; Rath, B.B. Study of annealing twins in FCC metals and alloys. Metall. Trans. A 1990, 21, 2891-2896. [CrossRef]

19. Hu, H.; Smith, C.S. The formation of low-energy interfaces during grain growth in alpha and alpha-beta brasses. Acta Metall. 1956, 4, 638-646. [CrossRef]

20. Lin, B.; Jin, Y.; Hefferan, C.M.; Li, S.F.; Lind, J.; Suter, R.M.; Bernacki, M.; Bozzolo, N.; Rollett, A.D.; Rohrer, G.S. Observation of annealing twin nucleation at triple lines in nickel during grain growth. Acta Mater. 2015, 99, 63-68. [CrossRef]

21. Gleiter, H. The formation of annealing twins. Acta Metall. 1969, 17, 1421-1428. [CrossRef]

22. Bernard, P.; Bag, S.; Huang, K.; Logé, R.E. A two-site mean field model of discontinuous dynamic recrystallization. Mater. Sci. Eng. A 2011, 528, 7357-7367.

23. Hillert, M. On the theory of normal and abnormal grain growth. Acta Metall. 1965, 13, 227-238. [CrossRef]

24. Bystrzycki, J.; Przetakiewicz, W.; Kurzydłowski, K.J. Study of annealing twins and island grains in FCC alloy. Acta Metall. Mater. 1993, 41, 2639-2649. [CrossRef]

25. Song, K.H.; Chun, Y.B.; Hwang, S.K. Direct observation of annealing twin formation in a Pb-base alloy. Mater. Sci. Eng. A 2007, 454-455, 629-636. [CrossRef]

26. Bernacki, M.; Logé, R.E.; Coupez, T. Level set framework for the finite element modeling of recrystallization and grain growth in polycrystalline materials. Scr. Mater. 2011, 64, 525-528. [CrossRef]

27. Jin, Y.; Bozzolo, N.; Rollett, A.D.; Bernacki, M. 2D finite element modeling of misorientation dependent anisotropic grain growth in polycrystalline materials: Level set versus multi-phase-field method. Comput. Mater. Sci. 2015, 104, 108-123. [CrossRef]

28. Scholtes, B.; Shakoor, M.; Settefrati, A.; Bouchard, P.O.; Bozzolo, N.; Bernacki, M. New finite element developments for the full field modeling of microstructural evolutions using the level-set method. Comput. Mater. Sci. 2015, 109, 388-398. [CrossRef]

29. Saltykov, S.A. The determination of the size distribution of particles in an opaque material from a measurement of the size distribution of their sections. In Proceedings of the Second International Congress for Stereology, Chicago, IL, USA, 8-13 April 1967; pp. 163-173.

(C) 2015 by the authors; licensee MDPI, Basel, Switzerland. This article is an open access article distributed under the terms and conditions of the Creative Commons by Attribution (CC-BY) license (http:/ / creativecommons.org/licenses/by/4.0/). 